\title{
LUCA CAVALLI SFORZA E I GENI DEGLI EUROPEI
}

\author{
s.c. GUIDO BARBUJANI (*)
}

(Adunanza del 6 giugno 2019)

È davvero un motivo di orgoglio essere stato invitato a parlare di Luca Cavalli Sforza: un grande maestro ma anche un italiano di straordinaria intelligenza e di singolari capacità imprenditoriali, grande scienziato ma anche persona che è riuscita a cambiare il volto della ricerca in genetica promuovendo progetti di grande portata, e conducendoli al successo. So che alcuni dei presenti non hanno familiarità con il suo lavoro, e quindi parlerò rapidamente di alcuni dei campi di cui Cavalli Sforza si è occupato, per poi concentrarmi su un tema in cui ha dato un contributo fondamentale e su cui si continua a lavorare: i geni degli europei. Vi dirò anche che gli ultimi studi demoliscono il mito secondo il quale gli europei hanno, e hanno sempre avuto, la pelle bianca. Oggi sappiamo che non è così: lo studio del DNA antico ci dice che, fino a poche migliaia di anni fa, gli europei non erano quelli con la pelle bianca, come siamo abituati a pensare.

Cavalli Sforza è nato il 25 gennaio del 1922 ed è morto l'anno scorso a 96 anni, lasciando la propria impronta sulla ricerca genetica a livello mondiale. La prima cosa importante che vorrei ricordare risale agli anni '50, quando aveva trent'anni. Fu a Cambridge, dove si occupava di batteri: era laureato in Medicina e i batteri gli interessavano dal punto di vista clinico, ma era persona di singolare curiosità e dopo un po' cominciò a interessarsi a questioni relative ai batteri che hanno poco a che vedere con le malattie che questi provocano e molto invece

(*) Dipartimento di Scienze della Vita e Biotecnologie, Università degli Studi di Ferrara, Italy. E-mail: bjg@unife.it 
con l'evoluzione, cioè il tema di cui successivamente si è occupato in maniera prevalente. Ha trattato in particolare la sessualità dei batteri, un tema delicato dell'evoluzione. Negli anni Cinquanta si scopre che anche nei batteri ci sono delle forme di sessualità, cioè di scambio di pezzi di DNA. Una delle forme batteriche più abili in questo scambio fa parte della specie Escherichia coli, un ceppo di batteri che Cavalli Sforza individua e chiama $H f r$.

Ma i suoi contributi sono soprattutto legati allo studio della genetica umana. Negli anni '60 e '70 Cavalli Sforza comincia a rendersi conto che il paradigma darwiniano, in cui gli organismi sono variabili e si adattano all'ambiente perché i portatori di certe caratteristiche vantaggiose lasciano più discendenti, in realtà non spiega tutto. C'è infatti una componente fondamentale, che possiamo chiamare "caso", non mirata all'adattamento, che deriva da singoli eventi, unici e in un certo senso irripetibili, nella storia delle popolazioni. Può capitare ad esempio che ci sia una carestia e una popolazione si estingua o si restringa, mentre (altrove, o in un altro momento) può accadere che siano disponibili molte risorse e che quindi la popolazione si espanda. Cavalli Sforza è tra i primi a esplorare le conseguenze di questi fenomeni, che noi chiamiamo di "deriva genetica", sia lavorando in Africa con i Pigmei, su cui ha pubblicato molti lavori, sia lavorando in Val di Parma. Questo suo interesse lo porta, nel momento in cui ha inizio il grande progetto del Genoma Umano, a rendersi conto di un limite molto forte. Il genoma umano è il complesso del nostro DNA; negli anni '90, quando viene lanciato questo progetto di lettura e di studio complessivo del genoma umano, sembra già molto ambizioso proporsi di descrivere completamente un genoma, i sei miliardi di basi o nucleotidi che lo compongono. Ma il genoma di chi? Ognuno di noi è infatti diverso dagli altri. Chi dobbiamo quindi scegliere? Da un lato è chiaro che avere a disposizione un genoma letto completamente è meglio che non averne nessuno; dall'altro, è altrettanto chiaro che se vogliamo capire perché certe persone hanno la pressione alta e certe l'hanno bassa; perché a certe persone l'aspirina fa bene, ad altri non fa niente e ad altri addirittura fa male; perché c'è chi digerisce il lattosio e chi no; per rispondere a tante domande come questa dobbiamo fare dei confronti. Un solo genoma non basta, e Luca Cavalli Sforza interviene vigorosamente per promuovere lo sviluppo in parallelo di un altro progetto, il "Progetto della Diversità Genomica Umana". Ottiene di farlo finanziare, si costituisce a Parigi un centro nel quale vengono raccolte 
linee cellulari di 52 popolazioni di tutto il mondo. Linee cellulari significa cellule che continuano a riprodursi, per cui chiunque nell'ambito della genetica potrà nei decenni successivi accedere a questi campioni e studiarne un piccolo pezzo, e così contribuire alla conoscenza del grande mosaico della nostra diversità genomica. È interessante vedere la mappa di queste 52 popolazioni perché costituiscono anche una descrizione dei viaggi di Cavalli Sforza. Quasi tutti questi campioni, se non li ha portati a casa lui personalmente, se li è infatti procurati attraverso contatti di lavoro e, a volte, anche amicizie. Ci sono nove campioni del Pakistan; ce ne sono molti dell'Africa, il continente a cui si è dedicato di più; e ci sono tre importanti campioni italiani: i sardi, i toscani e i bergamaschi. Disponendo di un tale archivio, è possibile farsi tante domande: come mai siamo fatti così, come mai ci sono al nostro interno certe differenze? E, quando è evidente che non è stata solo la selezione a farci così, quando ci sono ottimi motivi per pensare che la selezione abbia sì giocato un ruolo, importante ma circoscritto; allora quello che resta è la traccia di eventi storici. Le nostre somiglianze e differenze sono il frutto delle migrazioni che hanno portato un piccolo gruppo di africani, che era stato per milioni di anni sul ciglio dell'estinzione (si stima che fossero qualche migliaio) ad espandersi a un certo punto con stupefacente rapidità, colonizzando tutto il pianeta e portando all'estinzione altre specie umane, fino a diffondersi dappertutto assieme ai nostri compagni di viaggio, cioè i topi, che con noi hanno colonizzato tutto il mondo.

Se si parla di migrazioni (oggi è ancora più evidente di quanto non lo fosse 20 anni fa) si finisce per parlare delle interazioni fra popolazioni che si muovono, e quindi di come guardiamo il nostro prossimo e di come lo classifichiamo. Quando l'ho conosciuto, Cavalli Sforza non pensava che valesse la pena mettere in discussione il concetto di razza umana. Abbiamo discusso parecchio su questo tema, ma quando si è convinto è partito con la sua formidabile capacità organizzativa e da qui è nata una serie di iniziative culminate non tanto in progetti scientifici specifici (la parte scientifica l'abbiamo fatta negli anni ' 90 e poi si è sostanzialmente conclusa) ma in una serie di libri molto importanti. I testi di Luca Cavalli-Sforza, spesso firmati insieme a Francesco CavalliSforza, hanno contribuito come pochi altri a diffondere una cultura biologica di base e a mettere in discussione alcuni paradigmi dell'antropologia classica, per esempio l'idea che l'umanità possa essere divisa in gruppi razziali distinti. Oggi tra gli scienziati che si occupano 
seriamente di questi argomenti non c'è più dubbio che nella nostra specie non esistano gruppi razziali distinti.

Vorrei adesso spendere alcune parole sullo studio degli europei, perché qui il lavoro di Luca Cavalli-Sforza porta a una svolta fondamentale. La genetica di popolazioni, fino agli anni '70, era un campo di studio interessante, con una base matematica molto forte, ma forse non molto divertente. Si trattava, sostanzialmente, di calcolare il rischio che due genitori sani, ma portatori di una malattia genetica, potessero trasmetterla ai figli. In quegli anni Cavalli Sforza si rende conto che nei nostri geni c'è ben di più: c'è un messaggio dal passato, la traccia di quello che è successo prima, il segno delle nostre vicende, delle nostre migrazioni e anche di fenomeni culturali che hanno determinato dei cambiamenti demografici. Nel 1978, sulla rivista Science, insieme ad Alberto Piazza e Paolo Menozzi, Luca Cavalli Sforza sviluppa il primo studio sistematico delle popolazioni europee. Il gruppo di Cavalli Sforza disponeva di alcuni software sofisticati, molto potenti per quell'epoca, fornitigli dalla Nato. Mi dicono che probabilmente gli americani, in quegli anni impegnati nella guerra del Vietnam, studiavano i geni dei vietnamiti per scoprire se sarebbe stato possibile costruire un'arma biologica che colpisse solo loro e non gli altri. Questo obiettivo si è poi rivelato implausibile, e oggi sappiamo che i DNA di tutti noi, compresi i vietnamiti, sono identici per oltre il 99\%. Quando i dati genetici raccolti e il software sono stati messi a loro disposizione, Menozzi, Piazza e Luca Cavalli-Sforza hanno sviluppato metodi matematici per ottenere un'immagine tridimensionale della variabilità genetica degli europei. Nelle mappe si usano il verde e il marrone per indicare, rispettivamente, montagne e pianure. Allo stesso modo, Cavalli-Sforza e i suoi collaboratori ottengono un'immagine sintetica di come variano 38 geni negli europei (Per inciso: all'epoca sembravano tantissimi, ma oggi si lavora su milioni di varianti del DNA). Nella mappa si vede bene che queste varianti genetiche non sono distribuite a caso: c'è un gradiente che parte da sud-est con le tinte più scure $\mathrm{e}$ diventa sempre più chiaro, a mano a mano che ci si sposta verso nordovest. Dunque, qualcosa ha messo in fila le caratteristiche genetiche degli europei; ma cosa? Cavalli Sforza è persona di grandissima cultura, abituato a frequentare ambienti anche lontani dal suo. Presto si rende conto che esistono dati archeologici che corrispondono fedelmente a questo andamento. Dalla nostra origine fino a 10.000 anni fa, quindi da 180.000 anni, Homo sapiens si è alimentato delle cose che trovava spon- 
taneamente in natura, dei frutti della caccia e della raccolta dei frutti delle piante. Cominciamo a produrre cibo solo da 10.000 anni, a concentrare semi, a produrre utensili per l'agricoltura, e contenitori di ceramica per conservare il cibo. Al complesso di reperti archeologici che rimandano all'attività agricola diamo il nome di neolitico, la nuova Età della Pietra. Se guardiamo le mappe che descrivono i più antichi ritrovamenti neolitici in Europa, troviamo che sono straordinariamente simili a quella che descrive le differenze dei nostri geni. Si tratta di una correlazione, il che vuol dire che due fenomeni procedono insieme, non necessariamente che uno causa l'altro. Ma fra gli archeologi si discute se la diffusione delle tecniche neolitiche, dal Vicino Oriente verso nord e ovest, sia frutto di scambi culturali o di una migrazione degli agricoltori. L'idea è che i manufatti neolitici possano essersi diffusi o perché qualcuno migrava e li portava con sé o perché venivano imitati o trasmessi attraverso il commercio; ma se il meccanismo fosse stato solo commerciale non ci sarebbero conseguenze a livello genetico. Se scambiate il telefonino con un cinese, il cinese continua ad avere i geni dei cinesi e voi i vostri. Dal momento che la diversità genetica europea forma un gradiente che abbraccia tutto il continente, la spiegazione più logica è che ci sia stata una grande migrazione che ha prodotto il fenomeno. Solo l'ipotesi della migrazione, non quella che siano stati contatti e scambi culturali a diffondere l'agricoltura, spiega perché la distribuzione dei geni si sovrapponga a quella della diffusione dell'agricoltura.

Si chiama modello di Diffusione demica neolitica: i primi agricoltori del Vicino Oriente si sono gradualmente diffusi, portando con sé le proprie tecnologie agricole, i propri geni (e forse qualcos'altro, le loro lingue Indo-Europee, a cui accennerò più avanti). Però in realtà ho semplificato troppo, e adesso cerco di rimediare. La migrazione, in sé e per sé, non basta a creare gradienti. Negli ultimi trent'anni, molti romeni sono immigrati in Italia, ma non per questo abbiamo gradienti genetici che dalla Romania, attraverso Serbia, Bosnia e Croazia, arrivino in Italia. Perché si formino gradienti, e Cavalli-Sforza elabora il complesso modello matematico che lo dimostra insieme ad Albert Ammerman, bisogna che la popolazione che si diffonde abbia un potenziale di crescita superiore a quella che incontra nel corso della migrazione. Se è così; se le popolazioni che praticavano l'agricoltura disponevano di più cibo, e quindi potevano crescere più di quelle che continuavano a cacciare, allora i loro geni hanno potuto viaggiare e 
diluirsi poco alla volta, formando i gradienti che, 10mila anni dopo, Menozzi, Piazza e Cavalli-Sforza hanno identificato.

Dunque, ci sono vari motivi per credere che le caratteristiche genetiche degli europei odierni derivino da questo fenomeno di diffusione demica: un movimento migratorio proveniente dal Vicino Oriente. Di fatto, ci sono voluti anni per mettere d'accordo i tanti che, come archeologi o come genetisti, si sono occupati dell'evoluzione degli europei. Secondo Cavalli Sforza, c'è stata una prima espansione di popolazioni di cacciatori e raccoglitori, nel paleolitico. Venivano dall'Africa, erano nomadi, e hanno colonizzato (a basse densità, perché la caccia non forniva risorse sufficienti a crescere più di tanto) varie regioni dell'Europa. Poi, nel neolitico, lo sviluppo delle tecnologie per la produzione di cibo dà alle popolazioni del Vicino oriente, quella che a lungo si è chiamata la Mezzaluna fertile, un vantaggio decisivo; si espandono, si sovrappongono alle popolazioni preesistenti, in certi casi le sostituiscono. Oggi sappiamo che il modello di diffusione demica è quello che funziona meglio, ma ci siamo anche resi conto del fatto che non tutto era così semplice, che in diverse regioni d'Europa altre migrazioni, più localizzate, hanno avuto importanza.

La discussione interdisciplinare provocata dal modello di diffusione demica neolitica ha però messo in moto collaborazioni ancora più vaste. Nel 1988, insieme a Alberto Piazza, Paolo Menozzi e Joanna Mountain, Luca Cavalli-Sforza pubblica un articolo in cui compaiono due alberi che rappresentano graficamente le relazioni fra le popolazioni di tutto il mondo. Uno è un classico albero genetico, con le popolazioni dell'Eurasia, e poi delle Americhe e dell'Oceania, che si staccano dal tronco delle popolazioni africane; l'altro è un albero delle lingue parlate nel mondo. I due alberi a grandi linee si corrispondono, pur con una serie di eccezioni che andranno comprese: a maggiori differenze genetiche corrispondono maggiori differenze linguistiche. $\grave{E}$ nato tutto da un'intuizione di Charles Darwin, che nel suo libro del 1871, L'origine dell'nomo e la selezione sessuale, scrive appunto che la ricostruzione migliore delle relazioni linguistiche tra popolazioni potrebbe essere il loro albero evolutivo. In termini moderni, potremmo dire che la storia demografica dà forma alle differenze fra popolazioni, perché i processi che portano le popolazioni a diversificarsi (isolamento) o ad assomigliarsi (contatti) sono sostanzialmente gli stessi, sia a livello biologico, sia a livello culturale. Charles Darwin, come di consueto, ci aveva pensato prima degli altri. Solo negli anni Ottanta, però, prima 
nel laboratorio di Robert Sokal in cui all'epoca lavoravo, a Stony Brook, e poco dopo nel laboratorio di Stanford di Luca Cavalli-Sforza, si raccolgono i dati, linguistici e genetici, necessari per verificare se e quanto Darwin avesse avuto ragione.

L'ingresso di genetisti e antropologi, Robert Sokal e Luca CavalliSforza, nel dibattito sull'origine delle lingue non poteva non avere significative conseguenze. All'epoca, l'ipotesi tradizionale, dovuta soprattutto al lavoro di una linguista dell'epoca sovietica (lituana in realtà), Marija Gimbutas, era che le lingue IndoEuropee fossero arrivate in Europa dalle steppe dell'attuale Ucraina, intorno a 5.000 anni fa, nell'Età del Bronzo. Marija Gimbutas tracciava sulla mappa linee di diffusione delle lingue IndoEuropee, corrispondenti a possibili vie di migrazione, senza però disporre di alcun dato biologico. Si immaginava quindi un numero molto elevato di fenomeni migratori, ognuno dei quali aveva determinato la diffusione di una lingua. Un brillante archeologo inglese, Colin Renfrew, ha preso sul serio la teoria della diffusione demica. Si è chiesto quindi perché immaginarsi tante migrazioni dalle steppe, per nessuna delle quali era disponibile alcun dato biologico, quando ce n'era una, dimostrata da Cavalli-Sforza con convincenti dati genetici, che potrebbe spiegare come sono arrivati in Europa gli indoeuropei: non dall'Ucraina ma dal Vicino Oriente, e non nell'età del bronzo, ma nel neolitico. Renfrew propone che i primi contadini che hanno portato i propri geni e le proprie tecnologie in Europa, hanno portato anche le lingue. Si sono spostati a ovest dal Vicino Oriente, in Anatolia e poi nei Balcani e nel resto d'Europa, semplicemente perché a est c'erano altre popolazioni di contadini, che come loro stavano crescendo di numero e si sarebbero diffusi nel Nord Africa, in Arabia $\mathrm{e}$ in India.

Quello di Renfrew è un magnifico modello, in cui le informazioni provenienti da molti campi diversi - l'archeologia, la genetica, la linguistica - si combinano in maniera intellettualmente soddisfacente. Non ho tempo per entrare nei dettagli; posso dire che studi successivi l'hanno in buona parte confermato, ma anche dimostrato (e non è una sorpresa) che quando si studiano le popolazioni in particolare, o qualche aspetto del lessico e della sintassi, non sempre i conti tornano. Non c'è niente da fare: fenomeni complessi sono difficili da spiegare con cause semplici. Stiamo però vivendo in una fase in cui le conoscenze genetiche sono aumentate così tanto che possiamo porci domande fino a pochi anni fa fantascientifiche. Prima di entrare nell'ultima parte 
di questa chiacchierata, vorrei dire due parole sul nostro genoma. L'abbiamo già detto: chiamiamo genoma il complesso del nostro DNA. Il genoma è stato spesso confrontato metaforicamente con un testo: è il libretto delle istruzioni delle nostre cellule; come un testo, è costituito da lettere che noi sappiamo riconoscere, sono 4 molecole che indichiamo con le lettere A, C, T e G, che formano lunghissime catene, i cromosomi. Dunque, conosciamo l'alfabeto del DNA, e ne conosciamo anche il lessico, perché all'interno del genoma dei mammiferi ci sono circa 20.000 geni. Ognuno di loro produce una o più proteine e noi li conosciamo quasi tutti. Perché allora non riusciamo ancora a prevedere il cancro o il diabete o la pressione alta? Perché tante di queste caratteristiche - patologiche e no; vorremmo per esempio capire qualcosa delle basi biologiche delle nostre capacità fisiche e intellettuali - non dipendono da un solo gene ma da tantissimi; e quindi il contributo di ciascun gene in percentuale diventa molto piccolo, e difficile da riconoscere. Quindi, proseguendo con la metafora del testo, del genoma non conosciamo ancora la sintassi, cioè come i vari geni interagiscano tra loro e con vari fattori ambientali che li influenzano. Però è un testo che riusciamo a leggere ed è lunghissimo. Sono 6 miliardi e mezzo di caratteri all'interno di una cellula; secondo una mia stima, I promessi sposi sono lunghi un milione e mezzo di caratteri! Quindi il libretto di istruzioni delle nostre cellule è pari a 4000 volumi, ciascuno grande come I promessi sposi. Come faccia la cellula nel giro di secondi o minuti a trovare la riga giusta in questa biblioteca e fare la cosa giusta è un'altra cosa che ci sfugge e che in futuro ci piacerebbe imparare. Ma leggendo per intero questo genoma abbiamo capito, per esempio, che due persone prese a caso, una ragazza che viene dall'Asia e un signore nord-europeo, con il loro aspetto diverso, hanno in comune, in media, il 99,9\% del loro DNA. Quindi tutti i ragionamenti che facciamo noi evoluzionisti sulle differenze, fra individui e popolazioni, si concentrano su una frazione molto piccola del genoma, quella in cui troviamo delle differenze. Attenzione: in media significa che, in certi casi, queste differenze possono essere più piccole, e in altri più grandi. Il posto dove abbiamo trovato le differenze genetiche più grandi è l'Africa, il che conferma quello che ci dicono i fossili: l'Africa è un continente speciale, noi veniamo da lì.

Passerei ora a parlarvi del colore della pelle. Siamo abbastanza sicuri che, prima dell'agricoltura e della diffusione demica, l'Europa fosse abitata da cacciatori e raccoglitori che venivano anch'essi 
dall'Africa: le prime tracce fossili di uomini come noi, in Europa, risalgono a 45.000 anni fa. Quando sono arrivati, l'Europa non era spopolata: c'era l'Uomo di Neandertal, il vero europeo, da 300.000 anni. La nostra convivenza con loro non è durata a lungo: qualche millennio, poi siamo rimasti solo noi. Come abbiamo visto, intorno a 8.000 anni fa, dal Vicino Oriente, dalla mezzaluna fertile, arrivano i primi contadini, che nel giro di pochi millenni colonizzano tutta l'Europa. Inoltre, la Gimbutas qui aveva visto giusto, studiando le differenze nei genomi delle popolazioni europee, e anche qualche resto umano preistorico in cui il DNA si è conservato, si trovano tracce di migrazioni nell'Età del Bronzo provenienti dalle steppe pontiche. Dunque c'è un continuo sovrapporsi di strati: prima i Neandertal, poi i cacciatori-raccoglitori paleolitici, sopra di loro i contadini neolitici, e poi, soprattutto nel nord Europa, le popolazioni dell'Età del Bronzo.

Lo studio del DNA nelle ossa fossili non è banale, ma si stanno facendo grandi progressi. Una nuova prospettiva è quella di ricostruire caratteristiche fisiche di persone di cui sono rimaste solo le ossa fossili: il gruppo sanguigno, la capacità di digerire il latte. Il colore della pelle è un altro dei caratteri che dipendono da tanti geni contemporaneamente, e quindi non facile da studiare. I geni della pigmentazione sono attivi all'interno di cellule della pelle che si chiamano melanociti. I melanociti producono due pigmenti, le melanine: una più scura, l'eumelanina e un'altra più sul giallo, la feomelanina. Le melanine vengono concentrate in granuli che poi dai melanociti passano alle cellule più superficiali dell'epidermide: il particolare colore della pelle di ciascuno di noi dipende dal rapporto fra eumelanina e feomelanina nei granuli, da quanti granuli abbiamo, da quanto sono grossi. C'entrano parecchi geni e anche parecchi fattori ambientali perché, come sapete, quando ci si mette al sole ci si abbronza. Si tratta di un'interazione complessa che non abbiamo ancora del tutto decifrato, ma in cui cominciamo a orientarci. Ma come si è evoluto il colore della pelle? La pelle non lascia fossili, ma la maggiore esperta di queste cose, un'antropologa americana, Nina Jablonski, ci propone un modello, cioè un'ipotesi. Se aprite il pelo di un gorilla o di uno scimpanzé, la pelle sotto è bianca e quindi c'è motivo di ritenere che $\mathrm{i}$ nostri primi antenati africani avessero la pelle bianca. Per qualche motivo, quando ci separiamo dagli scimpanzè, forse sei milioni di anni fa, cominciamo a perdere pelo. A stare senza protezione sotto il cielo africano, con un'alta emissione di raggi ultravioletti è più facile sviluppare un tumore e quindi c'è una 
pressione darwiniana a sviluppare pelli sempre più scure. Quando compaiono le mutazioni che rendono la pelle più scura, queste mutazioni producono un vantaggio, un adattamento, e quindi si diffondono. A un certo punto, forse $70 \mathrm{mila}$ anni fa, qualcuno migra dall'Africa verso l'Asia e l'Europa. Nel nuovo ambiente, il meccanismo selettivo si inverte, perché alle basse esposizioni solari del nord Europa e del nord Asia con le pelli scure non si sviluppa abbastanza vitamina $D$, fondamentale nella gravidanza e nell'allattamento. Quindi nel nuovo ambiente diventa vantaggioso avere le pelli chiare, e le mutazioni che riducono la pigmentazione vengono promosse dalla selezione naturale e, attraverso le generazioni, si diffondono. Quando questo sia successo non lo sappiamo con esattezza; sembrerebbe, e a lungo è sembrato, logico, pensare che, siccome siamo in Europa da 70.000 anni o giù di lì, abbiamo avuto tutto il tempo perché le pelli si schiarissero. Negli articoli di Lina Jablonski non c'è una data precisa, ma le illustrazioni indicano che pensava che intorno a 50 mila anni fa europei e asiatici avessero già le pelli chiare.

Non è così, e ce l'ha dimostrato lo studio dei genomi. L'anno scorso si sono studiate le ossa, datate a 10mila anni fa, trovate in Inghilterra, in località Cheddar, in una palude. Appartenevano a un individuo che è stato, con ogni probabilità, ucciso, come dimostra il foro ritrovato nel suo cranio. Ė stato possibile studiare il suo DNA, e, con metodi di intelligenza artificiale usati dalle polizie scientifiche, ricostruire approssimativamente il suo aspetto. L'uomo di Cheddar aveva pelle scura e occhi celesti: una combinazione oggi insolita che però, evidentemente, era presente in Inghilterra 10.000 anni fa. Quando la notizia viene pubblicata dai giornali, ci si rende conto che in realtà non era la prima volta che ci si trovava di fronte a una osservazione del genere. Qualche anno prima, nel sito spagnolo di La Braña, era stato scoperto un individuo vissuto 7.000 anni fa, anch'egli con la pelle scura e gli occhi celesti. Finora sono stati studiati i genomi di quattro di questo periodo, il Mesolitico; tutti quanti hanno la pelle scura, e tre di loro occhi celesti. Una mia collaboratrice, Gloria Gonzales Fortes, sta utilizzando gli algoritmi di intelligenza artificiale con cui si sono studiate le pigmentazioni di questi quattro individui. Prima di tutto, abbiamo verificato il margine d'errore, analizzando campioni di cui conoscevamo il colore della pelle, e verificando se corrispondesse o meno a quella predetta dall'algoritmo. Possiamo dire che il margine di errore sperimentale è del $4 \%$. Studiando i genomi antichi disponibili, stiamo rendendoci conto che 
fino al Mesolitico, intorno a 10.000 anni fa, indipendentemente dalla latitudine, le pelli erano tutte quante scure o molto scure. In seguito, nel nord cominciavano a comparire occasionalmente pelli molto più chiare. All'inizio del Neolitico sono ancora comuni le pelli scure, ma in Spagna compaiono pelli più chiare che nel medio Neolitico diventano ancora più chiare. Infine, nell'Età del Bronzo, ormai, gli europei hanno tutti la pelle chiara. Ancora una volta, il Neolitico è il momento di snodo fondamentale. Le prime presenze di pelli bianche sono documentate nel Caucaso intorno a 12.000 anni fa; quindi, probabilmente sono stati i discendenti di queste popolazioni, nel Vicino Oriente e in Anatolia, a portare le pelli bianche in Europa. È interessante che proprio negli stessi giorni in cui usciva il lavoro sul Cheddar Man l'allora aspirante Presidente della Giunta lombarda, oggi Presidente, Attilio Fontana, avesse invocato misure contro l'immigrazione per proteggere la razza bianca. A quanto pare, invece, le pelli bianche sono arrivate in Europa proprio grazie a immigranti.

Tutto questo, e altro, lo possiamo dire perché 40 anni fa Luca Cavalli Sforza ha capito che dentro il DNA delle nostre cellule, che allora non si poteva quasi nemmeno studiare, c'erano tante storie interessanti, e ci ha dato i primi strumenti per decifrarle. Oggi la tecnologia ha fatto enormi progressi, ma è a Luca Cavalli-Sforza che dobbiamo essere grati, perché è lui che ci ha permesso di muovere i primi passi in una direzione che promette di rivelarci aspetti del nostro passato che altrimenti sarebbero rimasti sconosciuti. 
